\title{
An Attempt in Modelling Early Intervention in Autism Using Neural Networks
}

\author{
Andrew P. Papliński \\ Computer Science and Software Engineering \\ Monash University, Australia \\ Email: app@csse.monash.edu.au
}

\author{
Lennart Gustafsson \\ Computer Science and Electrical Engineering \\ Luleå University of Technology, Sweden \\ Email: Lennart.Gustafsson@sm.luth.se
}

\begin{abstract}
We present a solution to a problem of early intervention in autistic learning. This is an addition to our model of autism which is based on Kohonen Self-Organizing Maps extended with the Source Familiarity Filter and the Attention Shift Mechanism. In particular we study the feature map formation when attention shift is restricted by familiarity preference. The network learns the stimuli from the source with the lowest variability in great detail at the expense of the other source. The early intervention neural controller modifies the probabilities of presenting stimuli from a given source in response to the attention shift acceptance/rejection signals. ${ }^{1}$
\end{abstract}

\section{INTRODUCTION}

In this paper we present a solution to a problem of early intervention in autistic learning. This is a continuation of our work on modelling autism using artificial neural networks presented in [1], [2], [3], [4], [5], [6].

Autism is a developmental disorder with diagnostic criteria (DSM-IV, 1994 [7]) grouped in three basic categories, namely, impairments in social interaction and in verbal and nonverbal communication, and restricted repetitive and stereotyped patterns of behavior, interests, and activities. In our modelling we concentrate on the subcategory of the third category which deals with "encompassing preoccupation with one or more stereotyped and restricted patterns of interest". Kanner [8], the first to describe autism, considered an obsessive demand for sameness to be a cardinal feature of autism, which is clearly related to the above diagnostic criterion.

It is generally agreed that attention shifting is not normal in autism but the underlying cause is a matter of debate with two main hypotheses, a general attention shifting impairment [9], and attention shifting restricted by familiarity preference or novelty avoidance [10], [11], [12], [13]. A brief review of these hypotheses can be found in [1], [6].

In our earlier work on modelling autism [1], [2], [3], [4], [5], [6] where we examined the use of Kohonen SelfOrganizing Maps (SOMs) subjected to two-dimensional and multi-dimensional data we found that attention shifting restricted by familiarity preference (mode 3 learning) causes the feature map to learn the data from the source with the least variability in its data, whereas normal learning (mode 1), i.e. learning with attention shifts to that source which presents new data, resulted in maps which adapt to the data from both

\footnotetext{
${ }^{1}$ Proc. Int. Joint Conf. Neural Networks, Budapest, July 2004, pp.29-34
}

sources. Learning under general attention shifting impairment (mode 2) resulted in maps that were very similar to the maps obtained from normal learning.

In this work we consider the problem of early intervention in the mode 3 learning, with attention shifting restricted by familiarity preference, and demonstrate how to modify the probability of presenting stimuli from different sources in order to achieve the resulting maps similar to that as in the novelty seeking learning mode (mode 1).

\section{THE MODEL OF AUTISTIC LEARNING}

Our model of autistic learning is based on Kohonen SelfOrganizing Maps (SOMs) [14] extended with the Source Familiarity Filter and the Attention Shift Mechanism as illustrated in the block-diagram in Fig.1. Details of our model

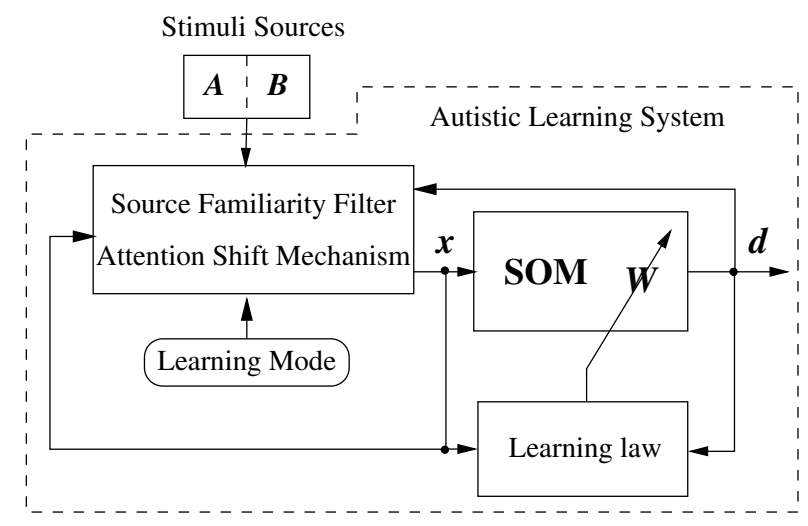

Fig. 1. A block-diagram of the model of autistic learning

were discussed in [1], [2], [3], [4], [5], [6]. Here we briefly present aspects of the model related to the early intervention problem.

The higher-dimensional stimuli are here chosen to represent animals arrange in two groups. One group (source A) contains animals of widely different kinds: mammals, birds, a reptile and fish. The other group (source B) contains only cats. Numbers of individuals in both groups are equal. There are animals which stand out and there are other animals that are very similar to each other, in some cases separated only by coloration. The animals are listed below:

Source A: Przewalski's horse, Grevy's zebra, canis lupus (wolf), dingo, white (mute) swan, black swan, Atlantic salmon, 
rainbow trout, polar bear, kodiak bear, white rhinoceros, hippopotamus, grey western kangaroo, swamp wallaby, anaconda, grey whale.

Source B: Even colored domestic cat, striped domestic cat, black panther, leopard, ocelot, jaguar, lion, tiger, black domestic cat, Siamese domestic cat, snow leopard, eurasian lynx, cheetah, cougar, fishing cat, serval.

The animals have been characterized by weight, food, locomotion (fins, wings, two legs, four legs), feet (hooves, claws or other), coloration (black, white, even colored, spotted, striped), facial feature (elongated or short nose), aquatic preference and social structure. Each animal is described by eighteen numbers. We project the animal characterization on a 19dimensional unity sphere. Hence, the width of the afferent connections in the neural network (dimensionality of the input space) is $p=19$. Arranging stimuli and weight vectors makes it possible to measure distances between them by the postsynaptic activities as described in [4], [5].

The neural network which is used to map the stimuli consists of sixteen neurons organized in a $4 \times 4$ two-dimensional grid. Due to the high dimensionality of the input space the feature map cannot be visualized in that space. However the dimensionality of the feature space, i.e. of the neuronal grid, is still low $(l=2)$ and the feature maps can be illustrated in this space by attaching to each neuron stimuli located in the proximity of the relevant weight vector.

At each learning step a stimulus is randomly generated from one of the sources, A or B. The Attention Shifting Mechanism determines if that stimulus is presented to the neural network for learning.

In the normal, i.e. novelty seeking learning mode (mode 1), attention is shifted to another source if the new stimulus originates from that source. In mode 2, general attention shifting impairment learning mode, attention is also shifted to another source if the new stimulus originates from that source, but only with the low probability of $1 \%$ (the results are insensitive to this number).

In mode 3, with the attention shifting restricted by familiarity preference, attention is shifted to another source if that source presents the next new stimulus, but conditionally, depending on the map familiarity with that source. The map familiarity to a particular source is measured by the time averaged value of the distance between map nodes and the stimuli. When both sources are unfamiliar to the map, i.e. in the beginning stage of self-organization, attention is shifted to an alternate source if that source presented the next stimulus as in the novelty seeking mode. As the map develops some familiarity with the sources, i.e. the node weights begin to resemble the data, attention is shifted with a higher probability to the source which is most familiar to the map. If the map becomes familiar to two or more sources then attention is unconditionally shifted.

We define the familiarity of the network with a source with the aid of a time average of the distances between stimuli and their respective best fitting neurons. The familiarity is changed each time the neural network has been exposed to a new stimulus. If the neural network learns one source well, the familiarity with that source will increase and reach a value close to one, which is defined as perfect familiarity.

\section{THE PROCESS OF LEARNING VISUALIZED}

The feature map resulting from the normal, novelty seeking learning is shown in Fig.2. The map consists of the $4 \times 4$

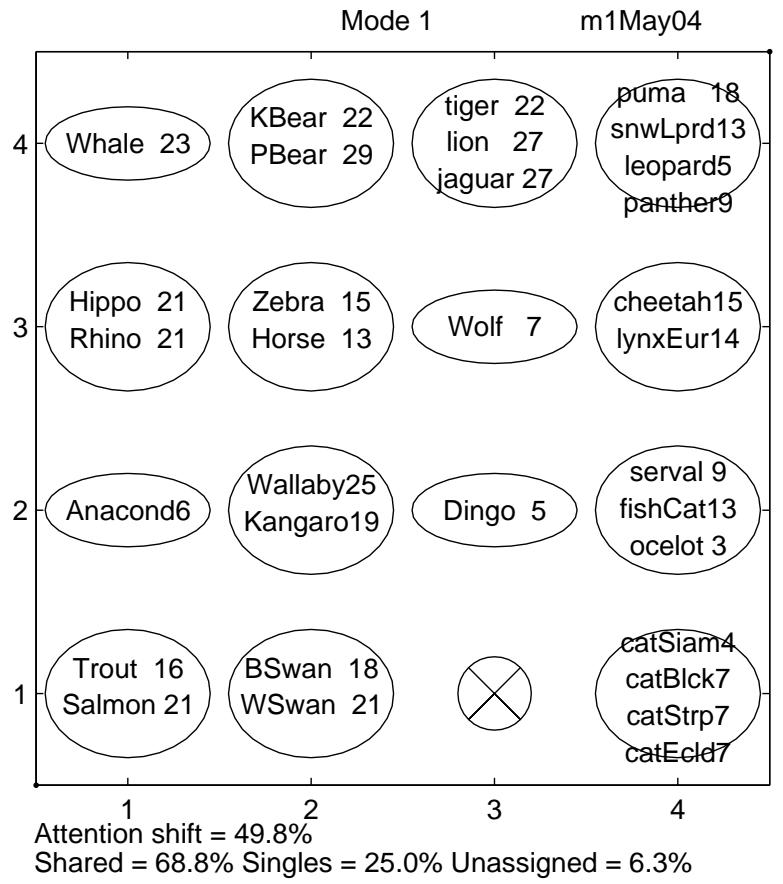

Fig. 2. The feature map developed in the novelty seeking learning mode.

neuronal grid. Each animal is shown at the node with the best match of weights. The angular distance between the animal vector and the node weight vector is shown after the animal name. In the map the number of animals for which a given node is the closest varies from four to zero ("dead neurons"). All animals are represented - the cats occupying five toprightmost nodes. One node is dead (unassigned).

In Fig. 3 we show how familiarity with the two sources grows in mode 1 learning. In this, as well as in all other simulations in this paper the initial synaptical weight values have been chosen to be equally distant from the stimuli from two sources. We see a steady increase in familiarity with both sources but the result will not reach one since the network does not have enough capacity to represent all stimuli (sixteen neurons cannot perfectly match thirty-two different stimuli).

The feature map resulting from learning with a general attention shifting impairment (mode 2) is shown in Fig.4. The map shows the same properties as the map resulting from the normal, novelty seeking learning (see [1], [2], [3], [4], [5], [6] for discussion and explanation). All animals but whale are represented in the map, and two nodes are unassigned.

In Fig.5 we show how familiarity with the two sources grows in mode 2 learning. In this case attention will be directed 

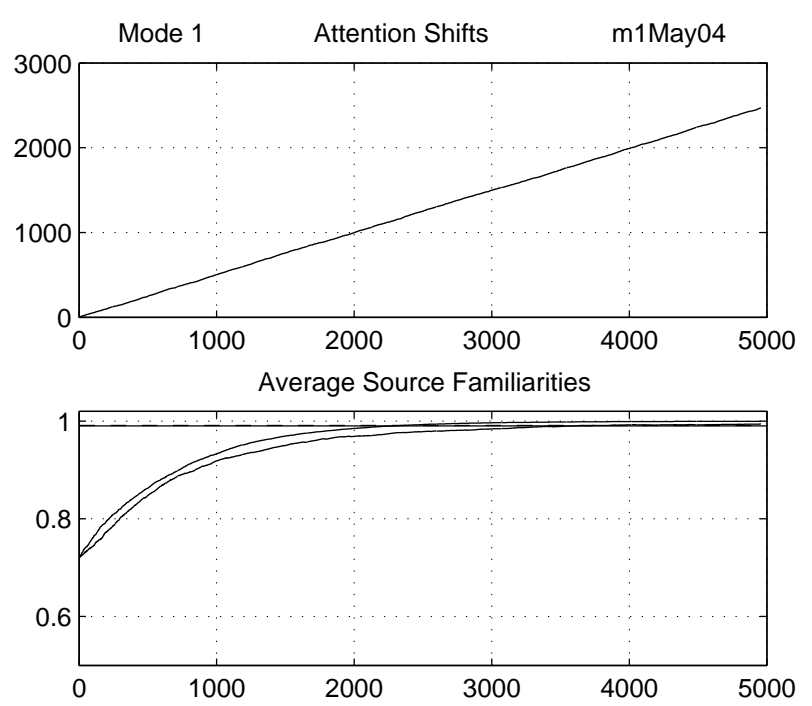

Fig. 3. Attention shift and the Average Source Familiarity for successive stimuli during the learning process in mode 1

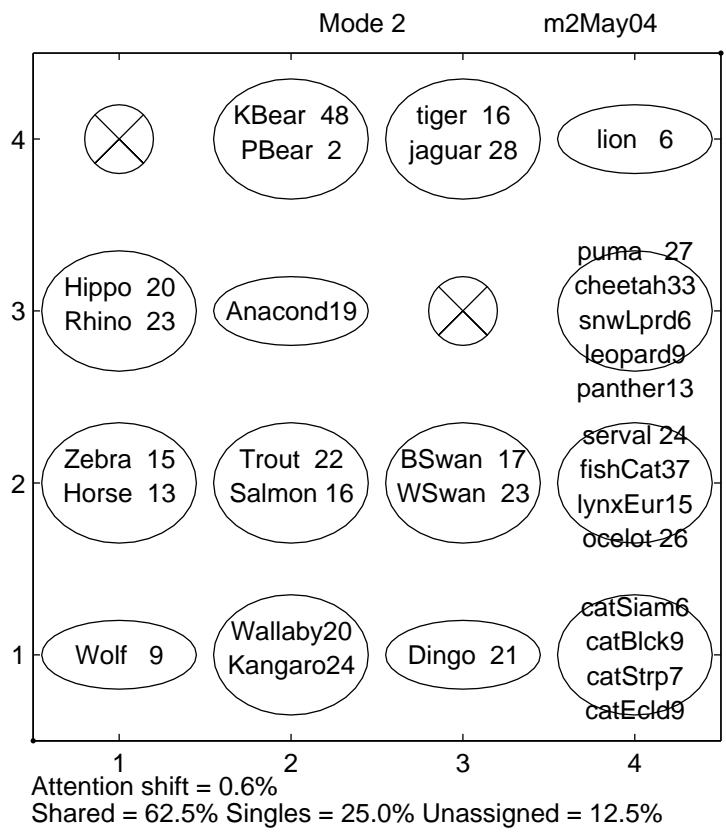

Fig. 4. The feature map developed in the mode 2 learning.

to one source for a long interval and learning of that source will proceed for that interval. After an attention shift the other source is likewise learned. We see that the familiarity with the sources of course develops in a more "chopped up" fashion but that the result is very similar to that of mode 1 learning.

The feature map resulting from learning in the attention shifting restricted by familiarity preference mode is shown in Fig.6. This map, which has developed largely after the attention shifting had ceased, reveals the characteristics of detailed learning in a narrow field, that is, the stimuli source
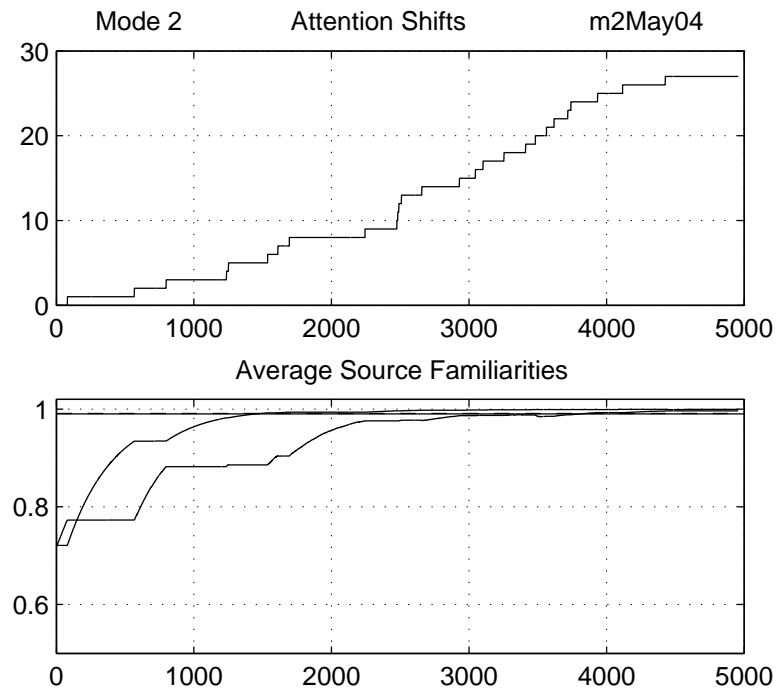

Fig. 5. Attention shift and the Average Source Familiarity for successive stimuli during the learning process in mode 2

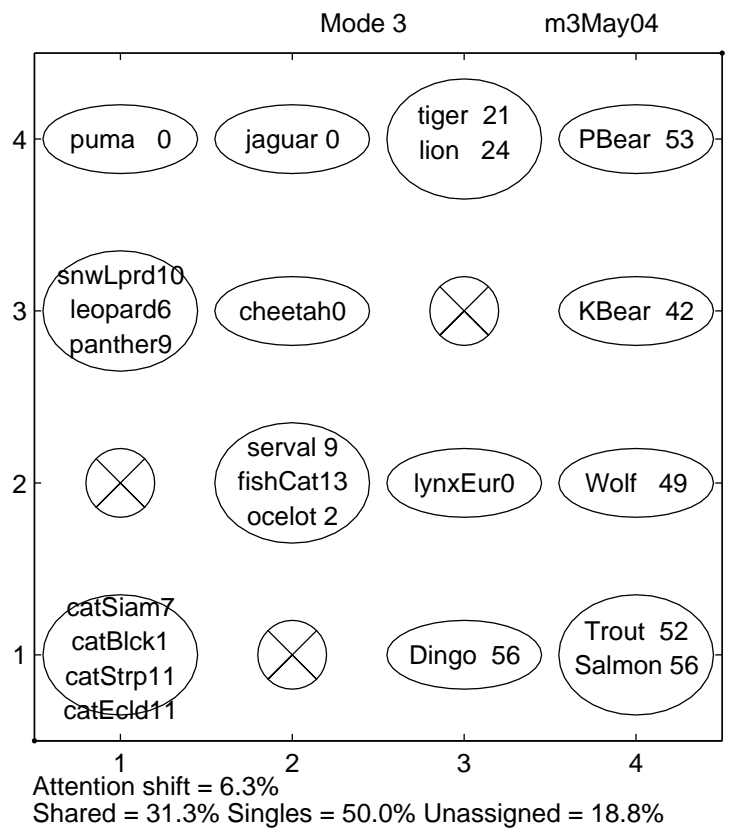

Fig. 6. The feature map developed in the mode 3 learning.

with the lowest variability, i.e. the cats, is learned. Every cat is represented by its best matching node and all best matches for a single cat are perfect. Three cat nodes give the best match for a group of closely related cats. Several of the nodes marked as "unassigned" have weight vectors which are close to the cats which have been allocated to neighboring (closest match) neurons. There is also a poor, "chance" learning of animals from source A.

In Fig.7 we show how the learning process proceeds in mode 3 learning. In this case there is an initial period when 

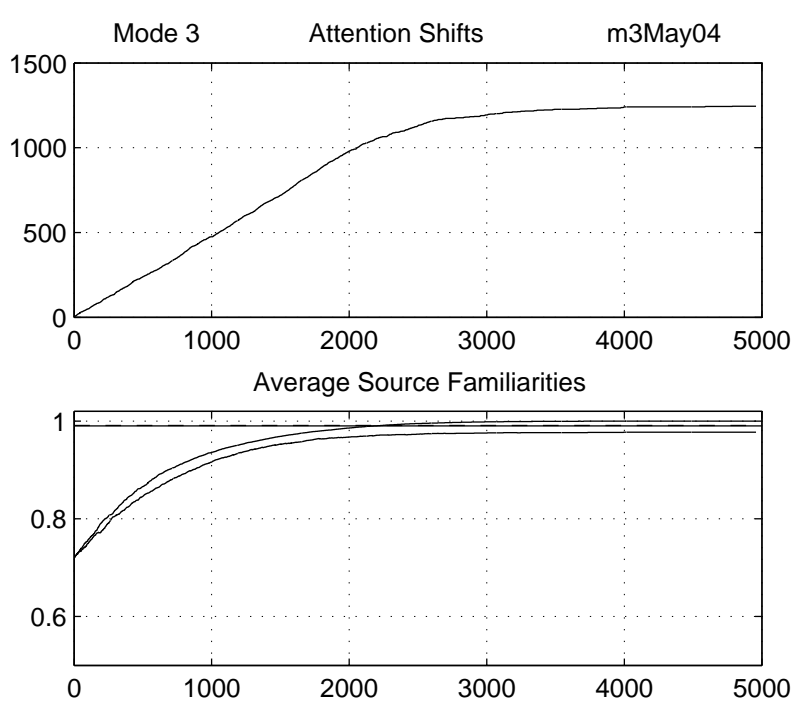

Fig. 7. Attention shifts and the Average Source Familiarity for successive stimuli during the learning process in mode 3

both sources are unfamiliar to the neural network and attention shifts occur just as in mode 1 learning. However as learning proceeds attention shifts to the most familiar source take place with a greater probability than to the least familiar source. After some time attention shifts cease to occur and attention is solely directed towards source B containing cats. In this case learning of that one source will yield a familiarity value of one or very close to one, whereas the familiarity with source A will be poor.

Relevant statistics of learning in all three modes were presented in [5].

\section{AN EARLY INTERVENTION SCHEME IN MODE 3 LEARNING}

It is obvious that source A, having more variability among its stimuli than source $\mathrm{B}$, demands more extensive learning than source $\mathrm{B}$ in order that the neural network will become equally familiar with source B and thus not reject attention shifts to source A.

If this is known a priori then of course presenting stimuli from Source A with a greater probability than stimuli from Source A would seem a reasonable scheme to explore. We will not here assume any prior knowledge about the variability among stimuli in the two sources. Instead we will attempt to adjust the probabilities of presenting stimuli from the two sources according to the attention shifts rejections, i.e. those instants when the next stimulus is presented by the source not attended to and attention shift does not occur.

A successful scheme for this change in probabilities follows. After a first attention shift rejection, which here is the first instant when an attention shift to source A is rejected, the probability of presenting stimuli from source A is drastically increased, very quickly to a value of 0.95 . Soon an attention shift to source A will be accepted and learning of source
A stimuli will proceed more rapidly than learning of source $\mathrm{B}$, simply because the neural network will be more exposed to source A. After some time this will result in a reversal of roles - source A will be more familiar to the neural network than source B and attention shifts to source B will be rejected. An opposite change in probabilities is now introduced and learning of source B will dominate and the degrees of familiarity of the neural network to the two sources will again be interchanged.

The two familiarity curves will be intertwined and this can go on for some more periods of changing probability but when the intervention has been successful - and this is almost always the case - both sources have become familiar to the neural network and attention shift rejections cease to occur.

Mode 3 learning with such early intervention is shown in Fig.8. After the first attention shift to source A (animals)
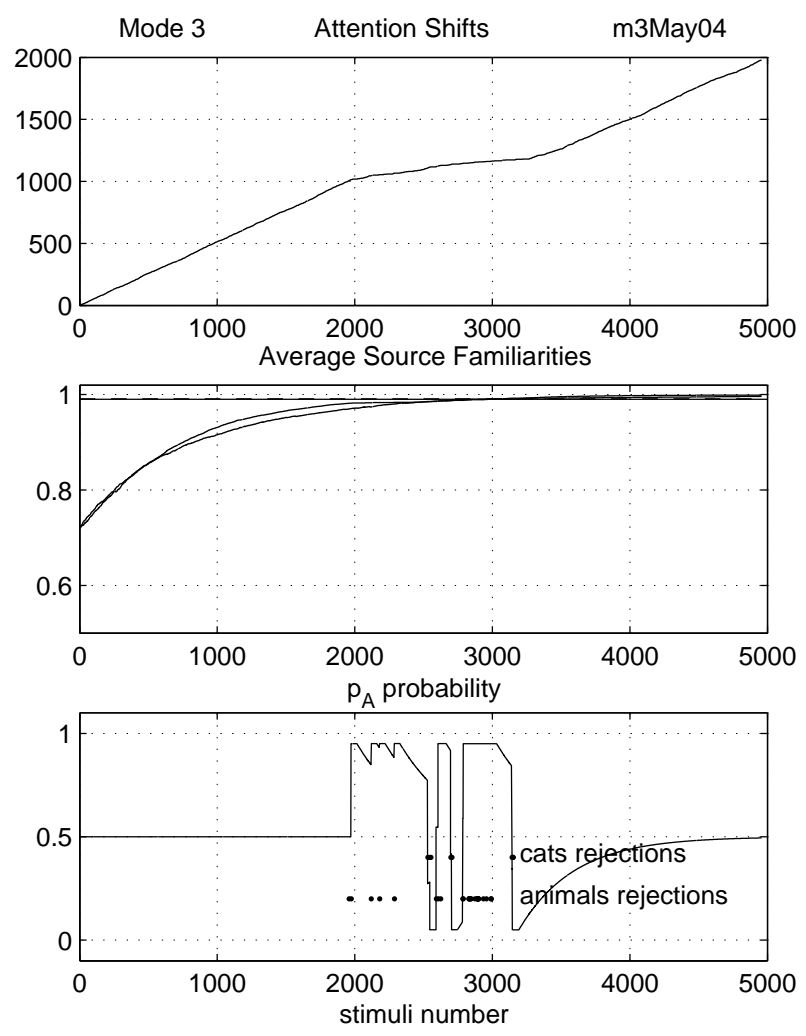

Fig. 8. Early intervention in learning in mode 3.

has been rejected, the probability $p_{A}$ for presenting stimuli from the source A is drastically increased. After a long period when the network is most often exposed to stimuli from the source A, this source will become more familiar to the network than stimuli from the source B and eventually an attention shift to the source B (cats) will be rejected. The probability for presenting stimuli from the source A is then drastically decreased. After this learning may have succeeded and several more attention shift rejection will occur and subsequent adjustment of the probability for presenting stimuli from the source A will be necessary. 
Note from Fig. 8 that the extreme values of the probability $p_{A}$ have been chosen. This is to achieve the fastest corrective action possible. Since a change in probability follows after a rejection of attention shift, it may be inferred that an unbalance in the familiarity of the neural network with two sources has already developed and it must be counteracted as forcibly as possible to bring the least familiar source back into play, i.e., back into the process of learning. Note also that the extreme values of the probability $p_{A}$ have been chosen close to 1 and 0 . If it is chosen exactly 1 , then stimuli from source $\mathrm{B}$ will not be presented ever and the neural network will not have its attention turned back to source B when this is appropriate. The reciprocal can be stated for choosing the probability as 0 . When the neural network has become familiar with both sources and no more attention shift rejection occur the probability is allowed to slowly change towards its "natural" value where all stimuli have an equal chance of being presented.

The resulting map, as is to be expected, has the same character as those for mode 1 and mode 2 learning as seen in Fig.9. All 32 animals are represented in the map.

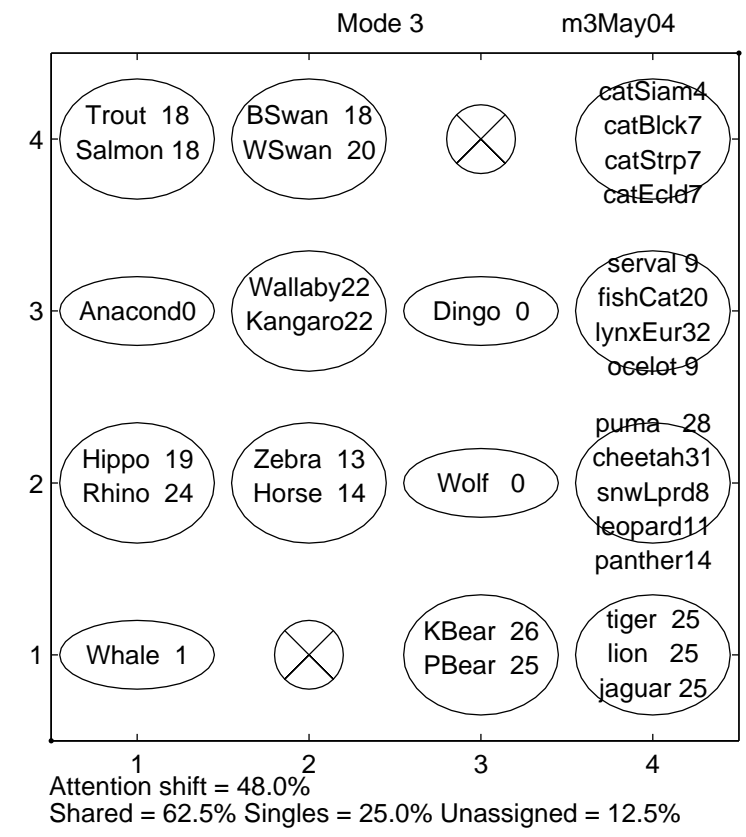

Fig. 9. The feature map developed in mode 3 learning with the early intervention process.

\section{SOME IMPLEMENTATION DETAILS}

The structure of an autistic learning system with an early intervention neural controller is presented in Fig.10. From the autistic learning system we are able to observe an attention shift to a specific source. We can say that the learning system generates four binary, mutually exclusive signals, namely, two acceptance signals, $a_{A}$ and $a_{B}$ - when the attention shift to a specified source is accepted, and two rejection signals, $r_{A}$

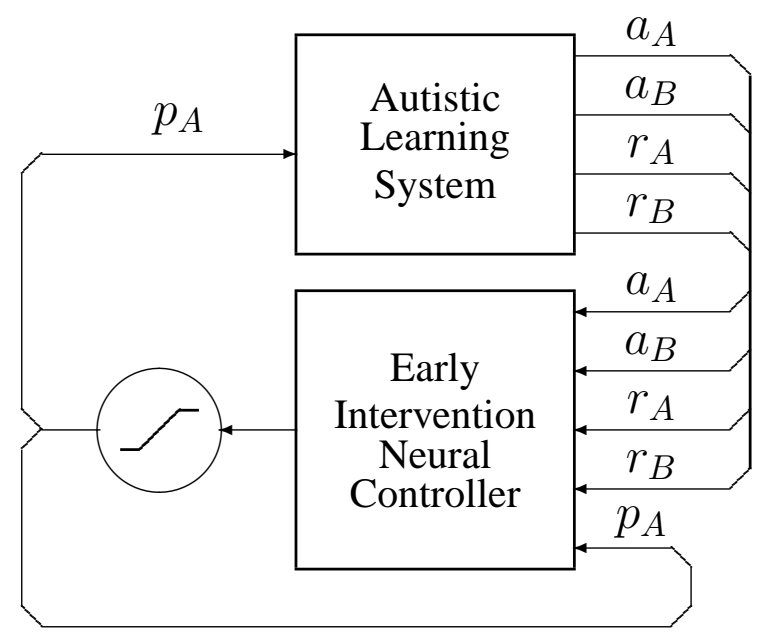

Fig. 10. The block-diagram of the interaction between the autistic learning system and the early intervention neural controller

and $r_{B}$ - when the attention shift to a specified source is rejected.

The only way we can externally influence the learning process is through the modification of the probability $p_{A}$ of presenting stimuli from the source $\mathrm{A}$, or, conversely, from the source $\mathrm{B},\left(p_{B}=1-p_{A}\right)$. The controller is a single neuron recurrent neural network with appropriately designed synaptic weights as illustrated in Fig.11. The probability $p_{A}$ is

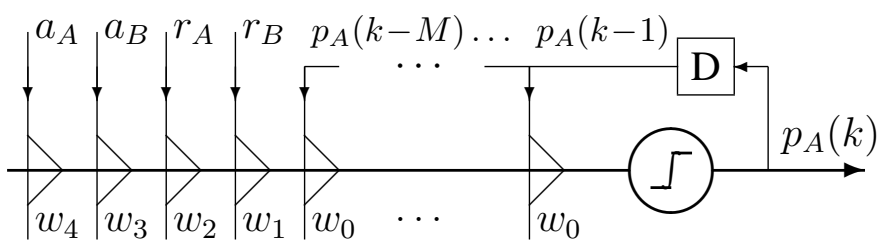

Fig. 11. The structure of the early intervention neural controller. Triangles represent synapses with weights as indicated. The block " $D$ " is the first delay unit of the tapped delay line which forms the past samples of the probability $p_{A}$

calculated as a capped linear combination of the four attention shift signals (moving average terms) and $M$ past values of probability $p_{A}(k-1), \ldots, p_{A}(k-M)$ (autoregressive terms).

\section{CONCLUSION}

We have presented a solution to a problem of early intervention in autistic learning. This is an addition to our model of autism which is based on Kohonen Self-Organizing Maps extended with the Source Familiarity Filter and the Attention Shift Mechanism. In particular we study the feature map formation when attention shift is restricted by familiarity preference. The network learns the stimuli from the source with the lowest variability in great detail at the expense of the other source. The early intervention neural controller modifies the probabilities of presenting stimuli from a given source in response to the attention shift acceptance/rejection signals. 


\section{ACKNOWLEDGMENT}

The authors acknowledge financial support from the Stiftelsen Einar och Ragnhild Lundströms minne, Sweden, and the Monash University, Australia.

\section{REFERENCES}

[1] Lennart Gustafsson and Andrew P. Papliński, "Attention shift impairments and novelty avoidance - effects of characteristics of autism on the self-organization of an artificial neural network," in Xth European Conference on Developmental Psychology, Uppsala, Sweden, August 2001.

[2] Andrew P. Papliński and Lennart Gustafsson, "An attempt in modelling autism using self-organizing maps," in Proc. 9th Intern. Conf. Neural Information Processing, Singapore, November 2002, pp. 301-304.

[3] Lennart Gustafsson and Andrew P. Papliński, "Autistic-like detailed learning in a narrow range of stimuli: results from simulations with artificial neural networks restricted by familiarity preference," in Inaugural World Autism Congress 2002, Melbourne, November 2002.

[4] Andrew P. Papliński and Lennart Gustafsson, "Detailed learning in narrow fields - towards a neural network model of autism," in Lecture Notes in Computer Science, O. Kaynak, E. Alpaydin, and L. Xu, Eds. 2003, vol. 2714, pp. 830-838, Springer-Verlag, ICONIP03.

[5] Lennart Gustafsson and Andrew P. Papliński, "Preoccupation with a restricted pattern of interest in modelling autistic learning," in Lecture Notes in Artificial Intelligence, V. Pallade, R. J. Howlett, and L. Jain, Eds. 2003, vol. 2774, PartII, pp. 1122-1129, Springer-Verlag, KES03.

[6] Lennart Gustafsson and Andrew P. Papliński, "Self-organization of an artificial neural network subjected to attention shift impairments and novelty avoidance: Implications for the development of autism," Journal of Autism and Developmental Disorder, vol. 34, no. 2, pp. 189-198, April 2004.
[7] "Diagnostic and statistical manual of mental disorders. 4th ed," American Psychiatric Association, 1994, Available from: http://www.psychologynet.org.

[8] L. Kanner, "Autistic disturbances of affective contact.," Nervous Child, , no. 2, pp. 217-250, 1943.

[9] E. Courchesne, J.P. Townsend, N.A. Akshoomoff, O. Saitoh, R. YeungCourchesne, A.J. Lincoln, H.E. James, R.H. Haas, L. Schreibman, and L. Lau, "Impairment in shifting attention in autistic and cerebellar patients," Behavioral Neuroscience, vol. 108, no. 5, pp. 848-865, 1994a.

[10] J.P. Kootz, B. Marinelli, and D.J. Cohen, "Modulation of response to environmental stimulation in autistic children," Journal of Autism and Developmental Disorders, vol. 12, no. 2, pp. 185-193, 1982.

[11] G. Dawson, A.N. Meltzoff, J. Osterling, J. Rinaldi, and E. Brown, "Children with autism fail to orient to naturally occurring social stimuli," Journal of Autism and Developmental Disorders, vol. 28, no. 6, pp. 479485, 1998

[12] D.M. Pascualvaca, B.D. Fantie, M. Papageorgiou, and A.F. Mirsky, "Attentional capacities in children with autism: Is there a general deficit in shifting focus?," Journal of Autism and Developmental Disorders, vol. 28, no. 6, pp. 467-478, 1998.

[13] N.J. Minshew, B. Luna, and J.A. Sweeney, "Oculomotor evidence for neocortical systems but not cerebellar dysfunction in autism," Neurology, no. 52, pp. 917-922, 1999.

[14] T. Kohonen, Self-Organising Maps, Springer-Verlag, Berlin, 3rd edition, 2001. 